\title{
Tangence
}

\section{Le téléphone d'Alexandre Grine}

Fuite et rumeurs dans la Russie révolutionnaire des années vingt

\section{Johanne Villeneuve}

Numéro 55, septembre 1997

La vitesse

URI : https://id.erudit.org/iderudit/025946ar

DOI : https://doi.org/10.7202/025946ar

Aller au sommaire du numéro

Éditeur(s)

Tangence

ISSN

0226-9554 (imprimé)

1710-0305 (numérique)

Découvrir la revue

Citer cet article

Villeneuve, J. (1997). Le téléphone d'Alexandre Grine : fuite et rumeurs dans la Russie révolutionnaire des années vingt. Tangence, (55), 40-63.

https://doi.org/10.7202/025946ar d'utilisation que vous pouvez consulter en ligne.

https://apropos.erudit.org/fr/usagers/politique-dutilisation/ 


\section{Le téléphone d'Alexandre Grine. Fuite et rumeurs dans la Russie révolutionnaire des années vingt}

Johanne Villeneuve

Aux lendemains de la révolution russe de l'automne 1917, Alexandre Grine - nom volontairement anglicisé par un écrivain admirateur de littérature européenne d'aventure et d'exotisme critique sévèrement ce qu'il considère être le "carnaval" furieux du dogmatisme et du fanatisme bolchévique ${ }^{1}$. Fervant sympathisant de l'anarchisme et du changement social durant l'été de 1917, il ne peut concilier le romantisme révolutionnaire de sa jeunesse avec la terreur bolchévique qui sévit alors. Entre la révolution et l'année 1921, la Russie est plongée dans le chaos le plus total. C'est l'expérience personnelle de Grine dans cette Russie révolutionnaire qui l'incite à écrire sa nouvelle "L'attrapeur de rats " ${ }^{2}$. Durant l'hiver de 1919, errant dans Petrograd, l'écrivain est en quête d'un gite et s'adresse à son ex-femme qui habite une chambre sans chauffage et souffre de la famine, comme la plupart des habitants de cette ville dont la splendeur et le faste architectural offrent un contraste étonnant avec l'indigence dans laquelle est tombée la majorité des citoyens. Petrograd, autrefois Saint-Petersbourg, coïncide plus que jamais avec sa réputation séculaire de "ville des spectres " ${ }^{3}$. Grine est obsédé par la recherche de nourriture; dans une lettre, il mentionne que sa maitresse

1 "Karnaval ", Oganëk, 1918.

2 "Krysolov", Revue Rossiia, 3, 1924.

3 le caractère fantomatique de la ville est présent dès sa fondation en 1703 du seul fait qu'elle fut tirée de l'imagination débridée de Pierre I.e Grand qui voulut voir émerger du néant une grande capitale européenne, Cité mirage édifiée sur les milliers de cadavres engloutis pendant sa construction. Comme surgic des marécages, contre toute raison, Saint-Petersbourg est construite d'après des modèles de prestige: Amsterdam et Venise ont inspiré le souverain qui fit appel aux architectes européens les plus raffinés. Ville de palais, de monuments et de canaux dont le miroitement et le brouillard ont confirmé sa vocation "fantastique", Saint-Petersbourg s'est vu attribuer la malédiction due à toute folie des grandeurs: Pierre le Grand figure l'antéchrist dont le rêve assassin hante la ville. Les eaux de la Moïka, serpentant à travers la cité des grandeurs, contaminant son sous-sol, produisent illusions, 
lui cache de la confiture dans un buffet fermé à clef. La conscience d'être devenu un parasite est à son plus fort ${ }^{4}$. Ironiquement, c'est l'Armée rouge qui le sauve en l'obligeant à s'enrôler à l'été 1919. Pendant quelques temps l'armée l'envoie au service des transmissions téléphoniques, près de Vitebsk. La téléphonie, avec ses réseaux, ses circuits quasi-magiques, laissera ses traces dans l'œuvre. Au printemps de l'année 1920, des problèmes de santé forcent son rapatriement à Petrograd où il contracte le typhus. Après plusieurs semaines de délire, Grine erre à nouveau dans une ville défigurée où l'odeur de la mort est partout. Gorki lui vient en aide et le réfère à la "Maison des Arts" établie sur les ruines d'une ancienne banque; de là l'écrivain peut constater les dégâts de la guerre civile pour laquelle on comptera en 1921 plus de 1200000 morts. La démographie de la Russie s'effondre en même temps que son économie et son industrie. La déroute économique pousse les dirigeants bolchéviques à ouvrir un espace de libéralisation. La N.E.P. (Nouvelle Politique Économique) inaugure donc en 1921 une période de tensions et de contradictions idéologiques mouvementée, accentuant la corruption et même le banditisme, mais inaugurant par ailleurs une période de liberté de pensée sans précédent. Arrivées à leur apogée, les esthétiques débridées et les manifestes les plus divers qui se sont arraché le titre d'avant-garde artistique depuis les années 1910 sont confrontés à terme au principe de réalité; l'art de propagande, le futurisme, l'anti-esthétique constructiviste, l'art d'agitation et le Proletkult pénètrent l'espace quotidien des villes entre 1921 et 1928; le théâtre expérimental explose littéralement dans le sillon de Meyerhold; le cinéma devient le medium révolutionnaire privilégié et la littérature ne cesse de se redéfinir à travers les moindres tensions idéologiques. Mais par-dessus tout, c'est à la faveur d'une projection du futur que les discours s'entrechoquent Au milieu du désastre démographique, tandis que la société toute entière retourne au troc et constate qu'elle a régressé sur le plan industriel et technique par rapport à ce qu'elle était en 1913, les grandes rumeurs des avant-gardes artistiques laissent entendre

duplicités, hallucinations. Les fonctionnaires qui déambulent sur les ponts et dans les rues de la ville sont poursuivis par les ombres du passé, parfois terrassés par leur double. Voir à ce sujet Le cavalier de bronze de Pouchkine, Le nez de Gogol, Le double de Dostöievski ou Petersbourg de Biely.

4 Voir sur cet épisode, Paul Castaing, "Grine et la révolution", Revue d'études slates, Paris, I.lll/2, 1981, p. 203. 
42

pour leur part que l'avenir est déjà là, que le "monde futur" s'est accompli dans le choc du présent et que le règne de la machine - celui de la "bio-mécanique", des poètes psycho-ingénieurs et de l'utopie cristalline - est advenu. Les poètes futuristes chantent la transfiguration révolutionnaire de la Russie en sacrifiant l'ancien symbolisme à la faveur des images chocs de machines extravagantes. Le théâtre se décompose en machineries de carton et les rêves d'architecture brisent les limites du concevable: lignes de fuite à l'horizontale comme à la verticale, tours vertigineuses, défi aux lois de l'équilibre et à la raison. Avions, automobiles, tracteurs, lampadaires, téléphones, chaînes de montage, fils électriques se substituent aux anciennes idoles et investissent jusqu'à la matérialité des objets quotidiens (tasses, assiettes, tissus arborant les nouveaux glyphes de la technique du futur ${ }^{5}$. Mais le tribut à payer au futur est lourd, et nombreux sont les artistes pour qui l'effusion révolutionnaire et la fascination pour la technique font remonter à la surface les vieilles eschatologies, les visions catastrophiques de l'histoire dont la Russie n'a jamais pu se détacher complètement. Du Front Gauche de l'Art (la Revue LEF), le poète futuriste Khlebnikov voit dans le profil de la grue mécanique se dessiner la figure d'un oiseau géant dévorant des gens. Les cheminées d'usine deviennent aussi pour lui les trompettes annonçant la fin du monde. Les objets sont aux commandes de la nouvelle société des hommes, mais le passé ressurgit, dissonant et scandaleux, au milieu des fureurs nouvelles, sous une forme archaïque, primitive, presqu'animale.

Mais sans doute la naïve fascination des premiers futuristes dans les années 1910 pour les appareils de vitesse, de production et de transmission se transforme-t-elle en angoisse partagée après la révolution, sous l'impact de la concrétisation de l'utopie. La réalité est féroce. En rompant de manière aussi cruelle avec le quotidien, la guerre civile a abrogé la fête poétique; elle a forcé l'imaginaire à revoir, sur une place publique jonchée d'affamés et de morts, ses rêves d'utopie.

Au moment où Grine publie son récit fantastique "L'Attrapeur de Rats" en 1924, la technopolis est encore à l'ordre du jour; mais les rêves sont embrouillés de visions cauchemardesques. Et

5 Les dessins de Rodchenko et Stepanova entre autres. Voil aussi les ceuvres et artéfacts dans le catalogue de l'exposition Velikaia Utopiia 1915-1932, Bentelli et Galart, Bern/Moscou, 1993. 
au milieu de ces visions, il arrive encore, et même souvent, que surgisse une épiphanie, un "miracle" ou simplement, le "rire" tonitruant (celui de Maïakovski par exemple) qui prouve bien qu'au-delà de la misère et des désordres quotidiens, le souffle d'une histoire en marche est encore présent. Toutes les inventions sont encore possibles, car le remuement du temps reste perceptible sous la couche nauséabonde des choses sacrifiées par la révolution. Parmi ces choses, il y a la mémoire.

C'est ce dont parle Pasternak dans une nouvelle étrange, à la fois polémique et hermétique, en cette même année 1924. "Les Voies Aériennes" raconte en effet, en deux tableaux narratifs, la déchirure entre un passé idéaliste, lumineux, et le présent révolutionnaire, hautement tragique. Dans le premier tableau, la voix prophétique du poète annonce une catastrophe à venir. Un marin venu rendre visite à un couple d'amis le jour où leur enfant est enlevé par des tziganes apprend qu'il est en fait le père de celuici. L'image d'une nuée, celle d'un train, tranche à toute vitesse à la fois l'horizon du texte et les destins des protagonistes. La nuée "promène son regard", elle est sujet de l'histoire; elle se cabre et se pose sur ses pattes de devant à la manière d'un cheval, animal apocalyptique. Le paysage hyperbolique se confond avec l'engin dont la vitesse fait en sorte que tout ce qui est décrit dans le texte semble contaminé par lui : le toit de la maison devient un disque éblouissant, une "planète métallique", et les "fusées d'eau " de la mer se confondent avec la "pluie de menus débris" dont l'éparpillement est l'effet d'une "tromperie" provoquée par le grondement du $\operatorname{train}^{6}$. La tromperie trouve son équivalent diégétique lorsque la mère de l'enfant retrouvé nie la paternité de l'ancien amant. Au second tableau, le texte nous transporte quinze ans plus tard, durant la guerre civile. L'enfant est maintenant un homme que les révolutionnaires ont arrêté et condamné à mort. La mère s'adresse à son ancien amant devenu révolutionnaire, métamorphosé par ses nouvelles fonctions et dans les mains duquel repose le sort du fils. Pasternak concentre l'action dans un bureau minable, exigu et obscur, dont le sol est couvert de détritus. Le révolutionnaire ne reconnait pas tout de suite la femme; celle-ci ne reconnaît pas non plus la nature exacte des lieux et confond l'épaisseur des détritus avec un tapis. Les visions sont

6 Voir la traduction française "Les voies aériennes" dans CEuvres, Paris, Gallimard, 1990, coll. "La Pléiade ", p. 455-466. 
44

brouillées. Mais le téléphone va surgir au milieu du dispositif tragique comme l'instrument prenant prosaiquement le relais des voies aériennes, ces voies quadrillant dorénavant l'espace utopique de la Russie. C'est au téléphone qu'en un dernier sursaut de reconnaissance envers son propre passé le révolutionnaire décide de parcourir la ville à la recherche du jeune homme condamné dont il pressent la fin toute proche. Au centre du dispositif tragique, le téléphone est un organe nodal, mais aussi paradoxal: organe de l'efficience révolutionnaire permettant de ratisser la ville et d'obtenir la vérité, il est aussi l'organe de l'impuissance, car prisonnier des détritus, au fond de son obscur cagibi, le révolutionnaire apprend sans recours aucun la mort de son enfant et la vérité sur son propre aveuglement:

Il avait compris que l'enfant de Lelia pouvait se dissimuler sous chacun des noms qu'il avait lus sur les papiers et, debout devant sa table, il téléphonait à tel et tel endroit, demandait tels et tels renseignements et, d'appel en appel, il s'enfonçait de plus en plus au coeur de la ville, au coeur de la nuit, jusqu'au moment où s'ouvrit, béant, l'abîme du dernier renseignement, définitif et véridique, celui-là.

Il regarda autour de lui. [...] Il ressentait une effroyable douleur dans les orbites et, quand il embrassait la pièce du regard, elle voguait devant lui tout en stalactites, tout en ruisseaux. ${ }^{7}$

Le tragique opère ici depuis le vertige d'une modernité dont l'issue est fatale. Le téléphone guide au coeur de la ville, au coeur de la nuit, toujours vers un abîme plus profond que le narrateur appelle Vérité. La reprise du motif tragique des yeux crevés par cette vérité conduit le lecteur vers une issue abyssale, archaïque, comme au centre de la caverne des origines (les stalactites) où la mesure de toute chose se perd. Le recours métaphorique aux ruisseaux, comme évocation de la multitude, de l'écoulement et de la fuite des choses, rejoint l'idée d'un évanouissement, d'une déliquescence des destinées. À la fin du récit, la mère éperdue s'écroule parmi les déchets et l'espace du bureau se dilue avec elle.

C'est à la même époque qu'Alexandre Grine compose une histoire dont le caractère étrange ne tient pas tant à sa filiation avec la tradition fantastique, qu'à la tension qui l'habite entre une fascination positive pour le modernisme technologique (sa vitesse,

7 Ibid., p. 466. 
son contenu novateur) et une fascination pour les aspects régressifs et négatifs de celui-ci (la réémergence à travers elle de vieux mythes et d'eschatologies diverses). Construite sur le modèle du cauchemar qui ne se termine jamais, l'histoire racontée par Grine procède par associations oniriques dans la plus pure veine fantastique. En partie autobiographique, puisqu'il y retrace ses pérégrinations post-révolutionnaires d'après le souvenir encore vif de la maladie, "L'attrapeur de rats" est l'histoire d'un homme affamé, sans attache, sans famille, sans lieu où véritablement s'installer, et qui erre dans Petrograd sans plus se soucier de lui-même, "ayant fait une croix sur le passé comme sur l'avenir" 8 . La tourmente révolutionnaire y apparaît dans sa version fatale, comme si les choses ouvraient toutes à préparer la mort. La révolution pénètre le récit de manière détournée, comme une force de changement impossible à nommer mais néanmoins présente - une atmosphère, une intensité. La vitesse va devenir le mode de ce changement et les rencontres que le héros fera au hasard des rues viendront entièrement déterminer l'intrigue dont le lecteur ne peut dire si elle débouche véritablement sur une résolution. L'explication la plus simple serait celle qui consiste à dire que le hérosnarrateur a rêvé tout ça. Mais c'est ici que commence une impossible herméneutique du texte, car la seule clef pour y entrer consiste en la disposition du lecteur à l'intensité qu'il offre: croisements, points de fuite, fugacité, vélocité, émission de bruits, fluidité et décomposition d'images, aiguillage des sens sur des voies démultipliées, l'histoire racontée par Grine est celle d'une lutte protéiforme pour la survie. Dans la Russie où tout s'est métamorphosé, où l'impensable est arrivé, Grine saisit l'occasion de décrire, non pas le changement historique ou quotidien, mais l'angoisse et l'excitation elles-mêmes, cette tension provoquée par le télescopage de l'utopie et de l'origine. Le rêve d'un futur glisse dans l'abîme, dans la fondation lointaine de la culture - et ce rêve n'est pas celui d'un seul individu, mais celui d'un organisme démesuré: une cabale, une société de conspirateurs, une foule, rêve d'un "paysage original, une contrée, un pays même".

Dans la tourmente révolutionnaire, l'espace social a perdu ses significations antérieures; toute signification, devrait-on dire, a

8 Alexandre Grine, L'attrapeur de rats, traduit du russe par Paul Castaing, Lausanne, L'Âge d'homme, 1972 , p. 13. Les citations suivies de numéros de pages entre parenthèses proviennent toutes de cette édition. 
46

perdu ses assises et ne peut désormais apparaître qu'en des instants fugitifs, au détour de parcours erratiques, car les lieux de la ville ont changé de fonctions, de même que les citoyens. Les identités permutent selon la réorganisation du pouvoir. À la recherche d'un gîte, sortant d'une longue maladie, le héros de Grine tombe sur une ancienne connaissance, un épicier chez qui il avait jadis contracté une dette. Le texte indique qu'il ne le reconnaît pas tout de suite, car l'épicier est devenu fonctionnaire. L'effet de la duplicité recoupe dans cette scène la nature double de la rencontre, son miroitement trompeur même, car le lecteur est conduit d'emblée sur une fausse piste:

À cette époque, une rencontre fortuite me conduisit à un gîte couteux. En flânant le long de la Moikka, distrait par le spectacle d'un pêcheur (un paysan muni d'un filet au bout d'une longue perche suivait avec précaution le quai de granit. Parfois il plongeait son engin dans l'eau et retirait une poignée de menu fretin), je rencontrai un épicier... [...] Je ne le reconnus pas immédiatement: plus de tablier, ni de chemise d'indienne à motifs turcs, ni barbe ni moustache. Mon épicier était vêtu sobrement d'un costume d'allure militaire... (p. 21)

Pourquoi cette longue description du pêcheur qui donne d'abord à penser que celui-ci est l'objet de la "rencontre fortuite" dont il s'agit? Bien sûr, le pêcheur retient l'attention du héros-narrateur en ce que le "menu fretin" a certainement quelque chose d'évocateur pour qui n'a pas mangé depuis des jours. Mais le désir d'introduire ce pêcheur dans le récit ne s'arrête pas là. Cette introduction permet à la narration d'accentuer une fois de plus le caractère double et hasardeux d'une réalité décrite par un héros évoluant dans un monde qu'on ne saurait distinguer du rêve. Plus encore, l'introduction de l'élément de l'eau dans le texte inaugure une isotopie importante. Élément de la fugacité, de la conductibilitê, de la fuite, du miroitement, l'eau est double: visqueuse ou fuyante, transparente ou opaque, elle oscille toujours entre le mouvement perpétuel et la constance du miroir. L'ambiguïté créée par la rencontre du pêcheur pousse le lecteur à croire, l'espace d'un moment, que le lieu douteux vers lequel on le conduit est la Moïka reconnue pour le brouillard qu'elle diffuse sur les nombreuses voies qui la traversent. Or il s'agit d'autre chose, soit d'une maison aux dimensions infinies, véritable labyrinthe, lieu central du récit dans lequel le héros va se perdre. Cette méprise entre l'eau et la Maison trouve son explication dans le caractère onirique du récit et fera métaphoriquement retour à plusieurs reprises dans la narra- 
tion. La maison labyrinthique, gîte temporaire où l'ancien épicier envoie donc le héros, est une ancienne banque. Il s'agit d'un lieu où "deux cent soixante pièces étaient calmes et vides comme l'eau d'un étang" (p. 22). Ce calme et ce vide sont trompeurs, car le récit nous apprend par la suite l'existence d'une société de conspirateurs à laquelle le héros devra échapper en une course effrénée. Microcosme de la ville chaotique, la Maison est décrite comme un espace illimité, dont la géographie semble s'étaler jusqu'à avaler les quartiers environnants. En ruines, apparemment abandonnée, mais débordante de ses restes ("un mélange omniprésent de papiers de toutes formes, de toutes destinations, de toutes couleurs déferlait en un véritable déluge", p. 24), elle semble investir la ville entière. Le déferlement annonce la catastrophe que confirme encore l'envahissement du papier qui "s'élève à l'assaut des murs". Puis “ l'inondation blanchâtre progressait de parquet en parquet alimentée par les cascades des armoires géantes". L'abandon même de l'endroit vibre donc, créant de surprenants mouvements, se chargeant des restes de destinations perdues.

C'est par l'isotopie de l'eau que le récit instaure le climat propice aux dédoublements, à la confusion, à l'angoisse; c'est à partir d'elle que le mouvement de la confusion va être canalisé en déferlements, que l'angoisse sera précipitée vers une expressivité de la vitesse.

Le récit se déplie au rythme des associations oniriques, selon une économie de la survie, car le héros a peur et a faim: exacerbation de l'urgence, quête effrénée d'un lieu où se terrer, obsession de nourriture, la course angoissante du héros est vécue comme une régression infinie. Pur corps de sensations, le héros hypocondriaque de Grine est traqué comme un rat, dans un monde "qui le regarde", comme dirait Lacan, plus qu'il n'est regardé; ce corps dont les battements - le bruit des pas retentissants - rythment le récit est lui-même à la poursuite d'êtres insaisissables dont seules les voix et les pas sont perceptibles. Ces êtres qu'on ne voit pas, mais dont on entend les manifestations diverses (bruits de fête, d'attroupements, chuchotements; appels, conversations furtives, respirations, frôlements) finissent par être désignés comme étant des "rats", une véritable société de rats menée par un des leurs appelé "le libérateur" "9.

9 Avec raison Marianne Gourg rectifie la traduction du terme russe employé par Grine (Izbavitel') en préférant l'affrancbisseur à celui de libérateur pour 
48

Devant une armoire énigmatique dont on apprend finalement qu'elle contient, au mépris de toute vraisemblance, un festin merveilleux, le héros se sent pris du désir incontrôlable d'en défoncer la porte. Lorsqu'enfin l'armoire livre son secret, la vision cède encore à l'isotopie de l'eau: "c'était comme si l'eau d'un baquet reçue de pleine volée m'avait coupé le souffle" (p. 33). L'impression du "souffle coupé " coïncide parfaitement avec la course folle dans laquelle le héros sera bientôt entraîné dans le labyrinthe.

Le récit n'est jamais clair sur la véritable nature de celui-ci. Les dimensions de l'édifice ne cessent de déborder et, désorienté, le héros dresse un inventaire de repères, de détails visuels et d'objets dont la mise en série, la répétition dans l'espace le désoriente à nouveau; pire encore, c'est vers sa propre mise en abyme qu'il s'achemine:

Imaginez un homme évoluant dans le reflet de deux miroirs placés face à face et qui répèteraient jusqu'à l'hébétude l'espace compris entre eux. Il ne manquait qu'une chose: que sa propre image apparût dans l'encadrement d'une porte voisine. (p. 25)

L'onirisme engage l'espace, force tout point de fuite, à correspondre à l'intensité de l'angoisse vécue par le héros dont les réflexions "filtraient comme l'eau contenue d'un barrage" (p. 35). Ainsi sommes-nous à la fois à l'intérieur de la Maison et dans la tête fiévreuse du malade. Lorsqu'il trouve enfin la sortie du labyrinthe, il poursuit sa course folle à travers Petrograd, mais la ville est devenue à son tour une annexe du labyrinthe, et les rencontres qu'il y fait confirment que tout conspire contre son désir ${ }^{10}$.

S'il est de multiples pistes d'analyse pour cette nouvelle, il faut suivre l'isotopie de l'eau pour y reconnaitre la valeur d'une conductibilité narrative. La narrativité se déploie en des réseaux inattendus. Le récit semble se briser par le moyen de détours

tant choisi par Paul Castaing (Marianne Gourg, "Essai d'interprétation de la nouvelle d'A. Grin "L'Attrapeur de rats" ", Revue d'études slaves, Paris, LIX/4, 1987, p. 796). Contrairement à Osvoboditel' (libérateur), Izbavitel' renvoie au contexte sacré. Par ailleurs, l'interprétation que fait Marianne Gourg de la nouvelle est pertinente, mais s'arrête plus particulièrement sur son contenu psychanalytique. L'importance du téléphone n'y est aucunement soulignée.

10 Les deux personnages rencontrés sur son chemin sont alors identifiés comme de faux adjuvants: d'abord un jeune enfant abandonné, au secours duquel se porte le héros, se révèle être un imposteur; puis une ennemie se présente ensuite à lui sous les traits de la jeune fille qu'il recherche. 
brusques, mais toujours selon des associations établies entre un cerveau malade, celui d'un affamé, brûlé de désir, et le chronotope labyrinthique de la ville métamorphosée dont on n'arrive pas à saisir si elle a complètement échappé au temps ou si, au contraire, elle s'y est engloutie. De quel temps et de quel espace en effet s'agit-il? Du temps archaïque de la prédation? Du lieu où de mystérieux conspirateurs préparent la fin du monde? La boucle de l'obsession se boucle ici: c'est à la fois d'êtres invisibles, insaisissables que le héros a peur et de lui-même, puisque tous sont condamnés à la condition de rat.

Traditionnellement, comme le rappelle Wendy B. Faris ${ }^{11}$, le labyrinthe est le lieu d'exclusion des êtres hostiles. Le sentiment d'exclusion auquel ne peut se soustraire à son tour le héros de Grine lui vient de ce que tout a changé et qu'il persiste pour sa part à être, tout en ne sachant précisément pas qui il est. Personnage sans liens, qui n'a nulle part où aller, il se retrouve au milieu du labyrinthe comme en sa propre mémoire - mémoire terriblement déserte et pourtant bruyante et pleine de choses abandonnées. Le passé poussiéreux, disloqué, en apparence inerte, y semble mêlé à ce que Walter Benjamin appelait des a rêves d'avénir" "2: lustres de bronze, faïence, cuivre, abat-jour en forme de cloche mêlés aux armatures d'acier, boules de verre, rouleaux de câbles. Dans une des grandes pièces de cette ancienne banque "on aurait pu [y] organiser un bal pour mille danseurs. Au centre s'élevait une fontaine dont les gargouilles aux mufles béants, grimaçants ou tragiques, semblaient autant de têtes humaines" (p. 27). $\mathrm{Au}$ vertige des lieux se superpose le vertige des temps. La métamorphose des "têtes humaines" se concrétisera quelques pages plus loin, alors que de mystérieux conspirateurs tiennent une assemblée. Le héros, pétrifié et terré dans l'obscurité, les entend proclamer à l'unisson "Mort à l'Attrapeur de rats". Mais l'impression de catastrophe est d'abord inspirée des lieux mêmes, alors que le narrateur décrit, non pas ce qu'il a vu, mais ce qu'il croit reconnâ̂tre dans le vertige des temps. L'hallucination, sous le mode de l'épiphanie poétique, déborde alors, bien avant que l'on ne parle des conspirateurs; elle provient de la fascination même du héros:

11 Labyrintbs of Language: Symbolic Landscape and Narrative Design in Modern Fiction, Londres et Baltimore, John Hopkins University Press, 1988.

12 Je renvoie ici au livre sur les passages parisiens, en français: Paris, capitale du XIX siècle (Le livre des passages), Paris, Cerf, 1989. 
50

Je me mis au diapason de ce spectacle, j'assimilai son style. L'exaltation que procure la vue d'un énorme incendie me fut à nouveau compréhensible. La tentation de la destruction montait en moi comme une inspiration poétique: j'avais sous les yeux [...] tout un pays même. (p. 28)

L'hallucination produit alors, non pas l'image d'une foule, mais son passage:

Il était difficile d'imaginer qu'une foule de gens grouillait ici naguère [...] Sur toute chose s'était appesantie l'empreinte de la putréfaction et du silence. Une audace inouie était passée comme un souffle de porte en porte, cyclone formidable, indivisible qui s'était détourné aussi facilement que s'écrase une coquille sous le talon. (p. 28)

D'un seul trait, le récit fait coïncider l'image d'une foule indivisible et la force d'un courant traversant le labyrinthe (de porte en porte). La suite confirme l'isomorphie entre l'espace-temps et les circuits cérébraux du héros:

Ces impressions provoquaient une sorte de démangeaison cérébrale qui rendait fascinante l'idée d'une catastrophe, un peu comme ce magnétisme intérieur qui vous pousse à regarder dans le vide. Il semblait qu'en ces lieux une pensée unique répétée en écho enveloppât toute forme, et cette pensée opiniâtre comme un bourdonnement d'oreille, rappelait la devise: "tout est consommé - et se tait . (p. 28)

L'immense incendie dont on évoque la vision catastrophique sans pourtant que la vision ne soit jamais entièrement saisissable par la vue elle-même, comme si celle-ci hantait plutôt les lieux et les prédestinait tout à la fois, cet événement n'a pas d'autre scène que la "pensée" qu'il occupe - pensée ramifiée comme toute construction paranoïaque puisqu'il est dit qu'elle est unique et répétée en échos, qu'elle enveloppe toute forme. Grine réussit un tour de force poétique en démontant la logique du vertige révolutionnaire, en saisissant, au cour de l'événement catastrophique, tant les rêves d'avenir que les plongées abyssales dans le temps vers lesquels la révolution fait advenir la société. Comprenant trop bien la vélocité des choses de son temps, celle par laquelle le temps lui-même est soufflé par la déflagration d'un monde nouveau, Grine choisit de décrire les impulsions du quotidien en sacrifiant la vision au profit des bruits et de la vitesse. Les choses allant trop vite, on ne sait si elles changent vers l'amont ou vers l'aval, vers l'avenir ou vers un passé fantastique. La vue est ici 
sans conteste disqualifiée, car on ne peut logiquement voir ce qui passe si vite. La vitesse enfonce la perception au cour des incertitudes. Le seul souvenir prégnant demeure celui des bruits, des éclats, des fracas, des sons et des bourdonnements, puisque le visible se déplace à mesure et que la vision se perd dans d'inutiles répétitions. C'est pourquoi le récit multiplie les recours à l'ouïe dans un réseau d'images fantastiques où l'intensité du silence rend plus intenses encore les bruits. Avec Grine, nous baignons dans le son, pris dans le jeu des dimensions relatives comme le sont tous ceux qui, au milieu de l'obscurité, suivent avec anxiété les déplacements du proche et du lointain: frôlements, échos des pas, respirations "retentissent tout contre mon oreille si grand, si intense et saisissant était le silence qui régnait sur ce désert" (p. 24). Bruits d'orchestre, moindre son, bruit englué de pas, oreilles ennemies aux aguets transforment les lieux en espace organique, se confondent à la "substance médullaire de la Banque", à la "noire semence des chiffres", à la " cohorte fantomatique". Espace de la démesure où aboutissent mort et folie, la Banque est comparée à des lieux extravagants, lieux de silence absolu ou de fureur: Alaska et Niagara (p. 26).

La figure archaïque de l'bybris surgit au milieu des débris, hallucinante comme le reste, provoquant un parallèle scandaleux: ces lieux semés de détritus, habités par des spectres, renferment le rêve d'avenir. Le héros comprend soudainement qu'il s'y trouve un trésor, car tous les papiers, tous les restes déchus en ce lieu ne sont pas tombés en désuétude mais constituent plutôt la plus grande valeur. Déchéance n'est pas à confondre avec désuétude. Durant la guerre civile, sous l'effet de la pénurie de combustibles, la population s'est mise à brûler tout ce qu'elle pouvait trouver: papiers, livres, meubles, souvenirs... Les greniers étaient systématiquement pillés par les habitants des étages inférieurs. C'est d'ailleurs ce que Grine raconte au début de sa nouvelle, alors que son héros fouille les décombres anonymes et ramène dans son sous-sol, tel un rat, le combustible nécessaire pour ne pas mourir de froid. La destruction systématique du passé était alors devenue en Russie une affaire quotidienne, non pas qu'idéologique :

c'est que l'on pouvait extraire des centaines de chariots de ce papier d'emballage si estimé des commerçants, dans le dessein de vous voler sur le poids. De plus, les fils électriques, la ferraille représentaient plus d'une liasse d'assignats. Ce n'est pas 
52

sans raison que les fils et les prises avaient été arrachés sur presque tous les murs que j'avais examinés. Aussi, je ne pensais pas que l'épicier fût propriétaire du mystérieux entrepôt. Il devait avoir le sien en un autre endroit... (p. 37)

Les matériaux, objets, outils du quotidien prennent un sens détourné avec les transformations dramatiques que subit la société durant la guerre civile, et même après, soit durant la nouvelle politique économique entre 1921 et 1924. Dans la nouvelle de Grine, ils prennent même un sens ésotérique, magique et régressif, car l'idée d'un "trésor" est associée, ailleurs dans le texte, à la tradition mythologique des "brigands "13. Le détournement de la valeur permet d'introduire dans le récit la théorie de la conspiration: la gloire des profiteurs du passé se mêle à celle des profiteurs à venir. Le faste bourgeois renaît, travesti, défiguré, sous les traits fanatiques des conspirateurs révolutionnaires. De même, contre toute attente pour des esprits futuristes, les rêves d'avenir mobilisés ici sont grevés de passé, lourds de souvenirs, et la technophilie habituelle des avant-gardes révolutionnaires se transforme chez Grine en imagination fantastique, avec tous les relents que cela comporte. On ne se surprendra pas de voir le narrateur disserter sur L'atmospbère de Flammarion, sur l'étrangeté de l'orage électrique et des photographies que l'éclair imprime "sur le corps de sa victime" (p. 40). "À la moindre impression, raconte-t-il, une excitation inquiète semblable à l'éclair de magnésium au milieu des ténèbres, tendait mes nerfs comme une corde vibrant. "(p. 30)

Ce que le narrateur appelle ailleurs "le magnétisme intérieur" préfigure donc parfaitement un des épisodes les plus forts de la nouvelle, celui du téléphone. Le téléphone devient le dispositif de médiation par lequel le "cerveau malade" du héros et le chronotope chaotique se rejoignent. Organe vivant, annonciateur de mort, le téléphone rejoue microscopiquement, au même diapason, la trame contenue dans le labyrinthe, lui-même espace vivant, organique, plein d'une nature menaçante et belle à la fois,

13 Épisode de l'armoire magique contenant des victuailles et renfermant aussi des rats. Dans la mythologie des brigands, le caractère magique de ceux-ci est lié au fait qu'à chacun d'eux est associé un trésor. Si un paysan découvre sur ses terres un trésor enfoui, il doit prendre garde à ce que de mauvais esprits ne viennent le défier, car les brigands vengent toujours les trésors pilles. 
annonciateur de mort aussi puisqu'on y réclame celle de l'Attrapeur de rats.

La conductibilité devient le principe par lequel de l'isotopie de l'eau, le récit passe au téléphone - organe de toutes les médiations sociales, mais aussi, comme chez Pasternak, dispositif où se rejoignent toutes les trames, tous les circuits, tous les secrets de la ville. Mais chez Grine, le monde n'étant plus que le mauvais rêve d'un esprit dérangé, la magie du téléphone obéit à l'intensité des désirs et des peurs du rêveur. La conductibilité téléphonique trouve dans son rapport à la conductibilité de l'eau son principal contenu poétique: c'est l'expression de la vie ellemême, avec son mouvement, ses changements, ses courants conduisant à la fatalité.

Le téléphone est ici une machine psychique, un point de fuite où l'imaginaire s'engouffre. C'est un des thèmes courants de la littérature des années 1920 et même 1930 en U.R.S.S. que celui du blocage ou de l'avalement de l'imaginaire individuel par une symbolique et une projection collective trop forte, écrasante ou totalitaire, comme si le délire social était si puissamment construit, si efficace, qu'il finissait par violer l'espace intime, jusqu'à pénétrer dans la chambre du rêveur ${ }^{14}$. Mais ici, Grine conjugue les effets fantastiques traditionnels, le lyrisme romantique et l'influence d'un symbolisme à peine dépassé en Russie, à une excitation quasi prosaïque pour la technique du dispositif téléphonique. Or la technique, on le sait, a des sources lointaines, microscopiques, biologiques. Comme le mentionne Jean-François Lyotard, elle précède l'humain en ce qu'elle se rapporte à n'importe quel système filtrant de l'information utile à sa survie ${ }^{15}$. C'est en ce sens que Grine prend la technique au pied de la lettre, en s'appuyant sur son caractère systémique, en hypostasiant ce qui lie le téléphone à une économie de la survie. Comment sortir d'un espace dont on sait qu'il est sans issue? Voilà la question, le point de fuite qui se dessine à l'horizon du texte et

14 Voil par exemple les nouvelles écrites par Sigismund Krzyzanovski dans les années 1920-1930, ke cólèbre roman de Boulgakov Le maître et Marguerite, ou encore, l'imaginaire paranoïaque d'un peintre analyticue pourtant communiste pur et dur comme Pavel Fïlonov.

15 Jaan-Francois Lyotard, Linbumain: causeries sur le temps, Paris, Galilece, 1988. Dans la même veine post-heideggerienne, voir Avital Ronell, The Telephone Book, lincoln et Londres, University of Nebraska Press, 1989. 
54

sollicite l'intervention du téléphone. Non pas parce que celui-ci conduit à la sortie du labyrinthe, mais parce qu'il présente systémiquement, abstraitement, logiquement, le dispositif même du labyrinthe, dont use justement la narration. Il permet de saisir le fil de cette infinité de réseaux que le labyrinthe, lui-même microcosme de la ville, ne cesse de produire. La logique du récit repose sur une économie de la survie qui plonge un soi moderne clans une terrible circularité : vie et mort permutent sans cesse, peur et désir se renvoient l'un à l'autre au rythme d'un chronotope sur lequel aucune prise n'est possible. Le chronotope révolutionnaire repose en effet sur la détemporalisation et l'outrance spatiale - infinis déferlements de l'espace-temps en ruptures. Il est significatif qu'au moment d'évoquer le téléphone, le héros se trouve à occuper l'exacte position de ceux qu'il craint et abhorre: "une assemblée de viveurs ou de conspirateurs, à la recherche d'un lieu isolé et secret, peut-être même une puissante organisation connue et protégée par les comités de logement" (p. 38). Cette description d'ennemis potentiels correspond dans les faits à lui-même, car le héros, à la recherche d'un gîte où se cacher, habitant un lieu isolé, vient tout juste de piller le trésor contenu dans l'armoire. Avant de sombrer dans la fièvre, comme la plupart des habitants de Petrograd, il est obsédé par l'idée d'être "pris" en flagrant délit de voler. La crainte est liée aux bruits, à la promiscuité : "prêtant une oreille inquiète au bruit des loquets sur les places publiques: un visiteur attardé n'allait-il pas me surprendre? " (p. 15) Qui est le visiteur et qui est le voleur? Les rôles sont infiniment permutables dans ce récit. Rat craignant la société des rats, le héros agit dans le labyrinthe en profiteur, fantasmant à son tour sur la richesse potentielle des lieux, à la différence près qu'il agit seul.

C'est à ce moment précis qu'il se souvient de la jeune fille rencontrée par hasard, quelque temps auparavant, sur le marché noir ${ }^{16}$. Ils y vendaient tous deux des livres, se sont à peine parlés

16. Cette rencontre est d'ailleurs interrompue par l'arrivée de la police venue clisperser une foule qui enveloppe d'abord le héros et la jeune fille avant de les sćparer. Cette métaphore de l'enveloppement est d'autant plus significative ģue plus tard, une fois entré dans la Maison, le héros parlcra des "vastes espaces sonores [qui l']enveloppent. Ces espaces sonores l'amèneront à conclure à la présence de conspirateurs. Le pressentiment d'une foule dans le labyrinthe se conjugue alors à cette foule en rupture sur la place du marché. les deux présences sont illicites. 
- il ignore même son nom - mais dans un monde vidé de sa mémoire, la jeune fille a donné au héros deux souvenirs précieux pour la suite du récit: une épingle avec laquelle en attachant son col ouvert pour ne pas qu'il prenne froid elle s'est aussi attachée le héros; et son numéro de téléphone, inscrit sur une page du Quichotte et que le héros vendra par la suite par inadvertance. Outre la symbolique sexuelle de la scène, facile à décoder, et audelà du schéma du désir, la rencontre avec la jeune fille a une valeur particulière à l'égard du temps révolutionnaire. Le héros est un homme seul et sans mémoire, vivant à rebrousse-temps, tombé au milieu des choses comme au milieu de nulle part. La jeune fille devient pour lui la seule garante d'un devenir. Elle est signe d'avenir - d'un avenir paradoxalement toujours présent, horriblement présent dans le récit, voire immanent. Le héros emploie toute son énergie à essayer de la retrouver. Il veut, nous dit-on dans la nouvelle, lui faire part de sa découverte. Mais le lecteur se doute de la présence onirique constante de la jeune fille, comme si elle ne l'avait jamais quitté - ce que la scène du téléphone confirme implicitement. Seul contre tous, il cherche à reconstruire le seul lien qui lui reste. Or ce faisant, il entre à son tour dans le cercle de la conspiration. La suite du récit confirme cette double conspiration : les rats conspirent contre l'Attrapeur de rats, mais celui-ci, que le récit désignera ensuite comme étant le père de la jeune fille, est voué à la destruction sans merci du "Libérateur". Par d'extraordinaires coïncidences, tous les fils se rejoignent jusqu'à produire l'effet de réplication par lequel le héros ne cesse de voir se confondre la confirmation de ses appréhensions et celle de ses désirs.

Lorsqu'il lui vient à l'esprit de retrouver la jeune fille, n'ayant obtenu d'elle qu'un numéro de téléphone malencontreusement égaré, le héros de Grine se tourne néanmoins vers le téléphone. L'isotopie de l'eau revient cette fois pour confondre le numéro de téléphone, l'épingle et la conductibilité de la mémoire: "Cette jeune fille, dont j'ignorais jusqu'au nom, avait laissé en disparaissant une trace dont l'éclat rappelait celui de la vague qui déferle au couchant. Il lui avait suffi pour produire ce délicat effet d'une simple épingle.... (p. 39) La double articulation diégétique et métaphorique du souvenir est extrêmement cohérente dans le texte; elle atteint un niveau complexe de polysémie, mais reste toujours systémique: si le numéro de téléphone conduit à la jeune fille, l'épingle devient la clef de cette conductibilité. Comment le héros réussira-t-il à téléphoner, lui qui a oublié le 
numéro? De plus, "malgré l'abondance des téléphones dont l'un attirait involontairement mon regard, aucun ne fonctionnait" (p. 39). Quelques instants auparavant, il est significatif que le héros ait réussi à obtenir "un filet d'eau, bien maigre pourtant... signe de vie "d'un robinet défectueux. De la même manière, passant de l'isotopie de l'eau à la conductibilité téléphonique, le récit va accomplir le miracle d'une communication qui tient plus du magnétisme psychique que de la technique:

Je me persuadai que le numéro allait me revenir si je me mettais physiquement dans la situation de parler au téléphone. [...] En face de mon téléphone j'éprouvais ce pressentiment, cette étrange langueur, cette confusion de la conscience qui accompagnent la plupart des créatures absurdes de notre esprit... [...] j'étais semblable au fer devant l'aimant. Je décrochai. [...] étaitce un bourdonnement dans ma tête, une hallucination auditive? toujours est-il qu'en frémissant, je perçus un grésillement, la vibration même des lignes téléphoniques. [...] Dans l'appareil, comme il est de règle, l'espace visible vibrait, attendant le contact. J'étais la proie de visions troubles, aussi étranges que ce bourdonnement d'une ligne téléphonique dans une maison morte. Je voyais des enchevêtrements de fils arrachés par l'ouragan établir des liaisons dans des points inaccessibles de leur inextricable chaos, des gerbes d'étincelles jaillir des dos arqués de chats bondissant sur les toits, des éclairs magnétiques de tramways, la trame d'un tissu imprimé d'un anguleux dessin futuriste. Ces visions de l'esprit ne duraient pas plus qu'une palpitation de mon cœur qui se cabrait. Il cognait, traduisant dans son code indéchiffrable sa sensation des forces nocturnes. Alors par-delà les murs se leva, lumineuse comme la lune nouvelle, l'image de la jeune inconnue. [...] je guidais ma mémoire à travers une tempête de chiffres. (p. 39-42)

Tout est là : le contenu magique de la technologie, ses liens indélébiles avec toutes choses futures en ce qu'elle permet la prescience, la médiation entre les parasites auditifs et l'acouphène, la production d'images sonores, d'espaces invisibles. La comparaison entre l'espace téléphonique et la "maison morte" rend évidente l'esquisse d'une cartographie de l'espace social par la téléphonie ${ }^{17}$. On ne s'étonnera pas d'apprendre que de la

17 Plus loin, clurant l'étonnante conversation téléphonique qu'il réussit à avoir avec la jeune fille, le narrateur décrit la ligne qui se coupe en reprenant le parallc̀le avec l'espace du labyrinthe: "À nouveau la distance s'abattit entre nous comme un mur aveugle." (p. 46) 
même manière qu'il pénètre le réseau téléphonique par la seule magie de la volonté, le héros entre dans le labyrinthe avec pour seule clef un secret confié par l'épicier. L'épingle sert donc partout de clef en ce qu'elle rappelle quelque chose au souvenir du héros et provoque des courts-circuits entre le psychisme et la réalité, ouvre des pistes, instille de l'intrigue. L'épiphanie surgissant au milieu du trouble qui agite tant le héros, cette image de la jeune fille, est produite dans un espace-temps paradoxal: véritable vision auditive, elle s'accorde au déferlement chaọtique de la tempête en même temps qu'à l'instant d'une palpitation. Chez Grine, la fugacité donne prise à de longues descriptions baroques; la vitesse permet l'évocation de toutes les lenteurs, de tous les blocages et des retours les plus complexes: "Mais si nous gardions fidèlement la mémoire de toute chose, quelle raison supporterait impunément l'évocation instantanée de toute une vie, en particulier celle des sentiments passés?» (p. 42)

La raison ne supportant pas cela, c'est à la mort que le paradoxe conduit. Or, la mort prend dans le récit de Grine plusieurs formes. Il ne faudrait pas croire que Grine hésite tout simplement entre une isotopie romantique, celle de l'eau comme retour aux origines, et la technophilie (électricité, magnétisme, tramways, téléphone...). La tension entre la pulsion régressive et les rêves d'avenir indiquent plutôt une seule et même conductibilité liée à l'économie de la survie dans laquelle la différence entre la vie et la mort tient à peu de chose, où les revirements les plus brusques peuvent toujours se produire et surtout, où la mémoire prend une dimension singulière. Constatant avec stupéfaction que le standardiste répond bel et bien au téléphone, le héros voit les rouages d'une horloge se remettre en marche: "une main mystérieuse avait donné l'impulsion au balancier et le mécanisme à nouveau égrenait son tic-tac" (p. 43). Ce temps remis en marche ne peut produire qu'une fausse temporalité, un temps paranoïaque, dont la nécessité ne peut être poussée que par les sombres desseins des conspirateurs: temps de la mort, réactivé par la magie téléphonique, alors que le héros est mis en abyme (se tenant dans le labyrinthe, on peut dire qu'il se tient, immatériel, dans le téléphone même):

Alors, dans le hurlement des inductions électriques déchaînées se ruèrent, comme d'une porte brutalement ouverte, des voix cassantes rappelant le caquetage des gramophones. Des vociférateurs inconnus s'égosillaient dans ma main refermée sur 
l'écouteur. Ils se coupaient la parole avec la précipitation et l'acharnement de gens accourus au spectacle d'un fait divers. Les bribes de phrases rappelaient un concert de croassements: "ah, là là là là!» hurlait un être invisible, essayant de couvrir la voix d'un baryton [...] À travers cette cacophonie de bazar, faiblement, comme la vibration d'un moustique, perçaient des gémissements, une lamentation lointaine, des rires, des sanglots, des coups d'archet, le bruit de pas mesurés, un bruissement, un murmure. Où, dans quelle rue résonnaient ces paroles inquiètes, ces appels, ces exhortations et ces plaintes? (p. 43-44)

N'est-il pas étrange d'entendre sur les circuits téléphoniques la cacophonie d'une ville entière? Des voix fragmentées, précipitées, violentes le disputer à des voix se lamentant, à des bruits de pas, à des coups d'archet? Le bricolage des bruits, mise en scène furieuse de la rumeur sociale et des échos de mort qu'elle produit, trouve sa réplique exacte dans le labyrinthe. Plus tard, terré dans son coin, le héros entend en effet

rires, exclamations, chaises déplacées, bribes de conversations animées, claquements de portes. [...] C'était un banquet, un bal, un congrès, une réception, un jubilé. [...] Tandis que j'essayais d'en clémêler les particularités, les instruments attaquèrent un air de café-concert. (p. 57)

Le héros se trouve donc pris dans une version hyperbolique du téléphone, traqué par ces voix dont il aura à combattre les assauts répétés tout au long d'une course finale. La scène du téléphone s'achève encore sur ce parallèle entre le téléphone et la maison: la conversation avec la jeune fille se perd, parce que les mots prononcés par elle "s'éloignaient, se brouillaient et finirent par se confondre avec le crépitement de la pluie. Enfin la pulsation du courant devint à peine perceptible et je compris que c'était fini." Voilà une mort annoncée. Et justement: "La liaison était morte, l'appareil stupide et muetm. Depuis cette mort, la narration effectue un déplacement spectaculaire, recule, produit un zoom-out audacieux: "Devant moi, j'avais un mur, une boite, un récepteur. Au carreau tambourinait l'averse". Le transfert vient de s'effectuer entre la sonorité de la pluie dans le téléphone et celle de la pluie dans l'espace où se tient le héros: "C'était la nuit. [...] Je m'assis, soudain épuisé comme si j'avais gravi un rude escalier. Et pourtant je n'étais qu'au début de mon aventure. Elle commença par un bruit de pas lointains. " (p. 46-47)

La médiation est maintenant complétée entre le téléphone et la maison, sur des fils conducteurs permettant la résonance com- 
mune de la cacophonie, de l'espace et de la rumeur. La suite du récit nous entraîne, par effets d'accélération et d'abstraction, dans les dédales obscurs du labyrinthe, vers l'assemblée des conspirateurs où la mort de l'Attrapeur de rats sera proclamée. Il a d'abord "le pressentiment de l'inévitable rencontre" (p. 48). Puis, "mon pouls, suivant le rythme des pas, s'intensifiait ou s'interrompait, mais bientôt... mon cœur se mit à cogner à grands coups" (p. 48-49), Une voix de femme l'interpelle; il va vers elle sans la trouver, s'y précipite même, tandis que la voix se dédouble, se multiplie, se confond avec la sienne: "l'écho avait roulé mon exclamation en une rumeur sourde et confuse" (p. 51). Les dimensions se disloquent devant l'empressement des voix, leur exhortation à se hâter: "sans doute était-elle poussée par un désir ardent de me faire savoir ou de me montrer quelque chose d'urgent et le temps pressait" (p. 52), mais surtout: "ces mots semblaient avoir été prononcés tout près de moi [...] comme s'ils avaient été chuchotés tout contre mon oreille" (p. 51). L'expérience téléphonique, bien que terminée depuis longtemps, semble donc se poursuivre. Grine donne l'impression que toute communication téléphonique renvoie à l'angoisse d'une insoutenable proximité, car des voix parlent à distance mais tout contre l'oreille. L'imagination ne met pas longtemps à fabuler quelque chose comme des voix internes, des voix dont rien ne prouve qu'elles viennent de l'extérieur.

Cette inquiétude est portée tout au long du récit comme sur une crête dans le versant de laquelle le héros risque sans cesse de rouler et de s'abîmer. Comme à un rat, ces voix posent des pièges au héros saisi par "le ricanement glacé qui retentit de l'autre côté du piège" (p. 53).

L'étrange assemblée invisible est-elle composée de spectres, ombres du passé issues de la banque, contre-révolutionnaires, vampires, esprits qui divaguent? S'agit-il donc d'une assemblée des morts dans les décombres de l'histoire? "Je n'étais plus qu'une oreille et qu'un souffle. Mais j'avais l'impression que toute vie avait disparu de la terre...toute vie s'était retirée de ces lieux..." (p. 55), confie le narrateur. L'imaginaire téléphonique est ici contaminé par la morbidité des voix gramophoniques ${ }^{18}$. C'est

18 Parmi les "machines à parler", le gramophone est reconnu pour son caractère morbide en ce qu'il laisse entendre des voix au-delà de la mort pour en conserver le souvenir. Sur les machines à parler et de nombreux exemples 
60

que la fièvre traverse la narration toute entière, depuis le déclenchement de la maladie du narrateur, jusqu'à la fin du récit, le lecteur n'apprenant jamais quand s'arrête la lutte du malade contre la mort. Or le typhus, brouillant la vision du héros (et peut-être encore celle du narrateur), l'entraînant dans le flux sans limites de souvenirs et d'inventions, s'infiltre dans notre lecture comme un poison. Au début de son aventure, à l'hôpital où meurent bientôt cinq patients autour de lui, le héros se laisse happer par le dialogue des morts:

Dans mon délire, je croyais voir des visiteurs, des gens dont j'avais perdu la trace depuis déjà plusieurs années. J'avais avec eux de longues conversations et je priais chacun d'eux de m'apporter du lait aigre. (...) Cependant j'attendais secrètement que parmi leurs visages, flottant comme dans les vapeurs d'une étude, apparût celui d'une nouvelle infirmière qui devait être, ni plus ni moins, la jeune fille à l'épingle. (p. 18)

Voilà donc à quoi correspond l'épiphanie de tout à l'heure, ce visage de la jeune fille apparaissant, en toute présence, au milieu des bribes téléphoniques, parmi les voix des morts. Puis il ajoute: "et même lorsqu'elle ne se montrait pas, la salle toute palpitante de lumière voilée était emplie de sa présence invisible " (p. 18).

L'intrigue ne suit que les manifestations de cette présence dont on nous dit qu'elle est invisible mais qui revient sans cesse, confusément, depuis tous les circuits, tous les passages, tous les repaires et toutes les failles émaillant le récit. Plus la narration avance, plus l'intrigue se précipite. Les conspirateurs projetant de tuer le père de la jeune fille, soit l'Attrapeur de rats maintenant dévoilé, le héros tente de fuir à toutes jambes le labyrinthe. Il court sous l'emprise de la terrible rumeur que le lecteur est à nouveau invité à prendre pour la rumeur de la ville elle-même:

Impossible de fuir cette rumeur obstinée qui me rendait fou. Elle s'enflait ou diminuait par saccades comme le trafic d'une rue, parfois elle m'atteignait presque et je me repliais d'un bond derrière une porte, parfois elle me suivait d'une course parallèle, comme si elle préparait d'une minute à l'autre à couper ma retraite. (p. 61)

Que peut-on déduire de cette complexe mise en déroute, sinon qu'il s'agit pour le héros d'une tentative perpétuelle, effré-

littéraires, voir Franc Schuerewegen, $\grave{A}$ distance de voix, Lille, Presses Universitaires de Lille, 1994. 
née de se situer quelque part, d'évaluer sa localisation dans le temps et dans l'espace, d'arrêter un "ici, maintenant"? Arrivé au bout de sa course, après avoir fui la Maison, traversé la ville, échappé à des imposteurs et finalement, dans un geste surréel, bondi par-dessus le tablier mobile d'un pont ("j'avais entrevu le miroitement de l'eau au fond de la fente", p. 66), le héros aboutit à l'appartement où logent l'Attrapeur de rats et sa fille en confiant: "mais les circonstances de mon arrivée s'échappaient comme l'eau coule d'un poing serré, dès que je faisais un effort conscient pour les rétablir" (p. 67). L'isotopie de l'eau fait retour au moment où la déliquescence narrative est à son plus fort; le récit touche à sa fin; le rêve se défait; l'explication fantastique se précipite; puis l'auteur multiplie les pistes pour expliquer la teneur surnaturelle de son récit. Ce qui s'échappe comme l'eau qui coule d'un poing serré correspond à la situation du héros, à sa position dans l'espace-temps. Impossible de savoir s'il dort ou pas, s'il est sorti de son coma ou pas. L'intensité de la présence invisible est bien là, comme le poing fermé, mais le héros ne sait pas à quoi correspond l'ici-maintenant.

Cet ici-maintenant et cette identité en déroute, j'aimerais les relier à la fonction phatique si essentielle à la communication téléphonique. L'entière logique onirique proposée par Grine repose sur cette impossibilité du chronotope à se cristalliser, à se maintenir dans un dire phatique. Le vertige chronotopique est si grand (passage et précipitation d'un lieu dans un autre, d'une isotopie dans une autre, d'un temps dans un autre) qu'aucun je narratif n'arrive à saisir les dimensions et la localisation exacte d'un ici-maintenant. Pourtant, cet ici-maintenant prend toute la place et tout le temps du récit; mais au lieu d'entraîner un syntagme narratif, il provoque des déferlements d'images, de voix et de bruits dont la mise en syntagme est imposture. Il entraîne des paradigmes dont les repliements divers produisent des images poétiques, des épiphanies, mais surtout une présence. La nouvelle s'emploie à déverser le symbolique, à le dissoudre dans l'espace phatique de la rumeur. À cet égard, le téléphone est bel et bien l'organe central du récit, le coeur de la machine-labyrinthe, le noyau de la ville plongée dans l'intensité, dans la présence invisible de l'événement révolutionnaire par où fuient à la fois l'espace et le temps. Il est aussi organe de mort parce que le ici-maintenant dont il est question, étendant ses ramifications partout, se livrant à tous les déplacements, résonne des échos de l'absence. 
62

Partout de trop, le héros n'est personne (jamais nommé) et nulle part parce qu'il est partout (dans le labytrinthe en même temps que dans la ville, encore à l'hôpital, toujours chez la jeune fille et jamais avec elle, etc.).

Si Grine a choisi de broder autour de la légende du Roi des Rats, légende populaire en Russie à l'époque, c'est parce que la théorie de la conspiration y est explicitement projetée: le Libérateur, roi des rats, animal magique capable de métamorphoses, survient en temps de guerre et de famine, dénature les hommes, agit en nombre pour voler les honnêtes travailleurs, faire des profits, amasser des trésors, tuer et mettre le feu. L'Attrapeur de rats fournit au héros une explication dans la plus pure tradition des dénouements fantastiques: "vous étiez entouré de rats" (p. 71).

En 1924, à l'époque où les contre-révolutionnaires se confondent aux révolutionnaires, où la corruption et les profiteurs sont légions, où la famine sévit et les rats - les vrais - prolifèrent, la référence est de mise. Elle conduit même à certaines élucubrations idéologiques dangereuses et qui deviendront populaires à partir des années trente dans certains milieux en Europe et aux États-Unis, soit la croyance en une conspiration des Juifs, des banquiers et des communistes! Mais si le rat est en évidence dans le récit de Grine, c'est aussi parce qu'il représente l'animal social par excellence, celui par lequel la rumeur circule et s'étend. Animal des fuites, des souterrains, présage de mort, il est en temps de famine celui qui mange et qui est mangé à son tour; traditionnellement, le rat est aussi l'animal des retournements, car on dit qu'il transmet des maladies autant qu'il peut guérir. Animal magique, il se fait médiateur, transmetteur, communicateur et contaminateur. La légende du Roi des rats décrit celui-ci comme un immense rat à seize têtes - têtes parlantes mises en scène dans le téléphone de Grine.

Cette double valeur positive et négative, dont l'ambiguité n'échappe pas au récit complexe de Grine, se reconnaît dans la figure séculaire de l'Attrapeur de rats, figure populaire en Europe, particulièrement en Allemagne et dans les pays slaves. L'Attrapeur de rats est celui qui envoûte les rats grâce à sa musique; rassemblés autour de lui, les rats enchantés vont le suivre en-dehors de la ville. Or, si l'Attrapeur arrive à débarrasser la ville de son fléau, il n'en demeure pas moins suspect du fait qu'il réussit à envoûter le fléau lui-même. Il se trouve à présent à la tête de la cohorte. Lorsque les rats reviendront pulluler dans la ville, les habitants 
pourront croire que l'Attrapeur de rats est celui qui les aura libérés de son charme et essaimés dans la ville, devenant par ce geste le libérateur - celui qui libère la peste dans la communauté.

Au pouvoir de la légende, à sa fascination régressive, Grine associe donc le délire fantastique de l'époque révolutionnaire. Le chronotope de la révolution, délié et propulsé de manière exponentielle dans des séries d'associations oniriques, produit une narrativité dont seule la vitesse et le bruit résonnent, une histoire où se déplie dans l'urgence l'unique fonction pbatique du langage - fonction dont on peut dire en quelque sorte qu'elle est devenue "folle". La tension entre régression et rêve d'avenir trouve à se dire dans l'étroitesse de l'espace phatique; mais cet espace se dilate à son tour, produit des ondes, accentue ses échos. Le téléphone de Grine, organe de présence et d'intensité, ne tend pas à démontrer que la tourmente révolutionnaire, point d'extase de la modernité, tournant violent du siècle, a seulement fait advenir quelque chose (l'histoire par exemple); il inonde plutôt l'espace de spectres, fait ressurgir depuis les arrières-pays la voix des morts, le visage du souvenir, la tentation du meurtre et des sacrifices séculaires. 